\title{
Inflammation and Disruption of the Mucosal Architecture in Claudin-7-Deficient Mice
}

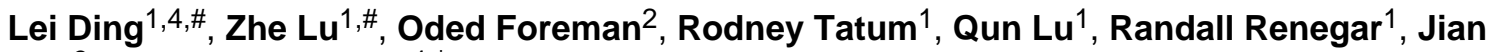 \\ $\mathrm{Cao}^{3}$, and Yan-Hua Chen ${ }^{1,}$, \\ ${ }^{1}$ Department of Anatomy and Cell Biology, East Carolina University Brody School of Medicine, \\ Greenville, NC 27834 \\ 2Department of Pathology, The Jackson Laboratory, Sacramento, CA 95838 \\ ${ }^{3}$ Department of Medicine and Pathology, Stony Brook University, Stony Brook, NY 11794 \\ ${ }^{4}$ Department of Oncology, Beijing Shijitan Hospital, Capital Medical University, Beijing, China
}

\begin{abstract}
Background \& Aims-Integrity of the intestinal epithelium is required for nutrition absorption and defense against pathogens. Claudins are cell adhesion molecules that localize at tight junctions (TJs); many are expressed in the intestinal tract, but little is known about their functions.

Claudin-7 is unique in that it has a stronger basolateral membrane distribution than other claudins, which localize primarily to apical TJs in the intestinal epithelium. We investigated the basolateral functions of claudin-7 and assessed the effects of disruption of $\mathrm{Cldn} 7 \mathrm{in}$ intestines of mice.
\end{abstract}

Methods-We generated $\mathrm{Cldn} 7-/-$ mice and examined their intestines by histology, molecular and cellular biology, and biochemistry approaches. We carried out gene silencing experiments in epithelial cell lines using small interfering (si)RNAs.

Results-The $C l d n 7-/-$ mice had severe intestinal defects that included mucosal ulcerations, epithelial cell sloughing, and inflammation. Intestines of $C l d n 7-/-$ mice produced significantly higher levels of cytokines, the NF- $\mathrm{KB}$ p65 subunit, and COX-2; they also upregulated expression of matrix metalloproteinases (MMPs)-3 and -7. siRNA in epithelial cell lines demonstrated that the increased expression of MMP-3 resulted directly from claudin-7 depletion, whereas that of MMP-7 resulted from inflammation. Electron microscopy analysis showed that intestines of Cldn7-/- mice had intercellular gaps below TJs and cell-matrix loosening. Deletion of $C l d n 7$ reduced expression and altered localization of the integrin $\alpha 2$ subunit; disrupted formation of complexes of claudin-7, integrin $\alpha 2$, and claudin- 1 that normally form in epithelial basolateral compartments of intestines.

(C) 2011 The American Gastroenterological Association. Published by Elsevier Inc. All rights reserved.

*Address correspondence to Yan-Hua Chen, Tel: (252) 744-1341; Fax: (252) 744-2850; cheny @ ecu.edu.

\# These authors contributed equally to the study

Conflicts of Interest Statement: All authors declare that no financial interest exists in relation to this submission.

Author contribution: Lei Ding and Zhe Lu were involved in the acquisition, statistical analysis, and interpretation of data as well as drafting of the manuscript. Oded Foreman characterized claudin-7-deficient mice and provided H\&E intestinal data. Rodney Tatum performed experiments, analysis and interpretation of data. Qun Lu provided technical and material support and was involved in experimental design and interpretation. Randall Renegar performed experiments and critically revised the manuscript for important intellectual content. Jian Cao provided technical and material support and critically revised the manuscript for important intellectual content. Yan-Hua Chen provided funding, the study concept, experimental design, study supervision, and finalized manuscript.

Publisher's Disclaimer: This is a PDF file of an unedited manuscript that has been accepted for publication. As a service to our customers we are providing this early version of the manuscript. The manuscript will undergo copyediting, typesetting, and review of the resulting proof before it is published in its final citable form. Please note that during the production process errors may be discovered which could affect the content, and all legal disclaimers that apply to the journal pertain. 
Conclusion-In mice, claudin-7 has non-TJ functions, including maintenance of epithelial cellmatrix interactions and intestinal homeostasis.

\section{Keywords}

Mucosal integrity; epithelial barrier; mouse model; permeability; IBD

\section{Background}

Disruption of intestinal epithelial tissue integrity leads to various intestinal disorders such as inflammatory bowel disease (IBD). ${ }^{1}$ Cell adhesion molecules play a fundamental role in maintaining intestinal epithelial integrity. ${ }^{2}$ Claudins are a family of cell adhesion molecules mainly localized at apical tight junctions (TJs). ${ }^{3}$ Among 24 claudin members in mammals, at least 10 of them are expressed in the mouse intestines. ${ }^{4}$ Although multiple claudins are expressed in the intestinal tract, their exact roles in the intestinal system remain largely unknown. Although several claudin gene-deficient mouse models, which include claudin-1, $-5,-11,-14,-15,-16$, and -19 , are currently available, none of these mouse models show IBD-like symptoms. The only claudin member reported to affect intestinal structure so far is claudin-15. Claudin-15-deficient mice formed megaintestines in which the upper small intestine was two times larger than the normal intestine, indicating the importance of claudin-15 in regulating normal-sized morphogenesis of the small intestine. ${ }^{4}$ Despite the lack of animal models in specific claudin-deficient mice, defective epithelial barrier function or increased intestinal permeability has been implicated in IBD. ${ }^{5}$ Moreover, altered expression and distribution of claudin-2, -5 , and -8 have been reported in biopsy samples from patients with Crohn's disease. ${ }^{6}$ However, whether the epithelial permeability defect observed is a cause or a consequence of IBD remains to be determined.

Studies have established that claudins are the most important structural and functional components of TJs and are crucial for the paracellular flux of ions and small molecules. ${ }^{7-8}$ Nevertheless, recent antibody-based studies indicated that several claudins, including claudin-7, are not only localized at apical TJs but also have a strong basolateral membrane distribution in the epithelia of various tissues, including intestines. ${ }^{9-11}$ It is essentially unknown what the functions of these non-TJ claudins are. In this study, using our claudin-7deficient mouse model, we discovered that claudin-7 interacts with integrin $\alpha 2$ at the epithelial basolateral compartment of intestines. Deletion of claudin-7 gene reduces integrin $\alpha 2$ expression and disrupts integrin $\alpha 2$ localization and claudin-7/integrin $\alpha 2 /$ claudin-1 complexes that formed in normal intestines, leading to MMPs upregulation, intestinal epithelial tissue damage, and invoking the inflammatory response, which resemble IBD-like symptoms.

\section{Materials and Methods}

\section{Antibodies}

Claudin-1, -2, and -4 antibodies were purchased from Invitrogen (Carlsbad, CA). Claudin-7 antibody was obtained from Immuno-Biological Laboratories (Japan). The antibodies against MMP-3, MMP-7, and MMP-8 were from Abcam (Cambridge, MA). Antimacrophage antibody CD11b (Mac-1) was from Miltenyi Biotech (Auburn, CA). Monoclonal and polyclonal antibodies against integrin $\alpha 2$ were from Chemicon (Millipore, MA) and Santa Cruz Biotechnology (Santa Cruz, CA), respectively. All other antibodies were from Cell Signaling Technology (Beverly, MA). 


\section{$\mathrm{Cldn7}^{-/-}$Mice}

The generation of $C l d n 7^{-1-}$ mice by gene targeting was described previously. ${ }^{12}$ The $C l d n 7^{-1-}$ mouse colony was housed in the microisolation barrier. To determine mouse genotypes, tail snips were obtained while newborn mice were tattooed. All animal experiments were approved by the East Carolina University Animal Care and Use Committee.

\section{RNA Isolation, PCRArrays, and Real-Time qRT-PCR}

Total RNA was isolated from $\mathrm{Cldn} 7^{+/+}$and $\mathrm{Cldn} 7^{-/-}$intestines, T84 and HCC827 cells using Qiagen RNeasy kit (Qiagen, Valencia, CA) following the manufacturer's instructions. PCRArray and real-time qRT-PCR experiments were performed using RT ${ }^{2}$ Profiler PCR Array system from SABiosciences (Qiagen). The relative changes in gene expression from real-time qRT-PCR experiments were analyzed using $2^{-\Delta \Delta C t}$ method. ${ }^{13}$

\section{Cell Culture and siRNA Transfection}

T84 intestinal and HCC827 lung epithelial cell lines were obtained from American Type Culture Collection (Rockville, MD). The cells were grown in DMEM/F-12 (T84) or RPMI 1640 (HCC827) medium supplemented with $100 \mathrm{U} / \mathrm{mL}$ penicillin, $100 \mu \mathrm{g} / \mathrm{mL}$ streptomycin, and $10 \%$ heat-inactivated fetal bovine serum. After reaching 70-80\% confluence, the cells were transfected with the specific siRNA against claudin-7 or with the scrambled siRNA (control) using Nucleofector solution and instrument (Lonza, Walkersville, MD). After $96 \mathrm{~h}$ transfection, the transfected cells and their culture media were collected separately for Western blotting analysis. The culture media were concentrated using the concentrator (Novagen, CA).

\section{Zymography}

$\mathrm{Cldn} 7^{+/+}$and $\mathrm{Cldn} 7^{-/-}$intestinal tissues were homogenized in RIPA Buffer and prepared in the sample buffer without reducing agent for electrophoresis. The equal amount of each protein sample was loaded and separated by $12 \%$ zymogram gel containing casein (Invitrogen). After electrophoresis, the SDS was removed from the gel by incubation in unbuffered Triton-X-100, followed by incubation in an digestion buffer (Invitrogen) for overnight at $37^{\circ} \mathrm{C}$. The gels were then stained with Coomassie Brilliant Blue and photographed using a Chemiluminescent Imaging with the Chemi DOC XRS (Bio-Rad, Hercules, CA).

\section{BrdU Injection and Detection}

$C l d n 7^{+/+}$and $C l d n 7^{-/-}$pups were intraperitoneally injected with BrdU (50 mg/kg, $1 \mathrm{mg} /$ $\mathrm{mL}$ ) dissolved in PBS. The pups were killed $2 \mathrm{~h}$ after injection. Swiss-rolled intestines were fixed in Carnoy's fixative ${ }^{14}$ overnight and embedded in paraffin. Sections were treated with $1 \mathrm{~N} \mathrm{HCl}$ at $60^{\circ} \mathrm{C}$ for $8 \mathrm{~min}$ to denature DNA. The slides were incubated with monoclonal anti-BrdU antibody (Sigma) diluted to 1:1000. The signal was detected by biotinylated antimouse IgG and peroxidase-conjugated strepavidin (Vector Laboratories, Burlingame, CA).

\section{Statistical analysis}

The data for real-time qRT-PCR assays were expressed as means \pm s.e.m. The differences between the two groups were analyzed using the unpaired Student's $t$-test. A $P$-value of < 0.05 was considered significant. 


\section{Results}

\section{Deletion of claudin-7 gene disrupted intestinal epithelial tissue integrity}

$C l d n 7^{-1-}$ mice were born viable and showed no obvious difference in appearance at birth (postnatal day 0, P0) (Fig. 1A, left). The appearance of intestines between $\mathrm{Cldn} 7^{+/+}$and Cldn $7^{-1-}$ was quite similar (Fig. 1A, right). However, the weight of one-week old $C l d n 7^{-1-}$ pup was only $40 \%$ of that of $C l d n 7^{+/+}$as shown in Fig. 1B. Bloody loose stools were always observed in $\mathrm{Cldn} 7^{-/-}$large intestine, but not in $\mathrm{Cldn} 7^{+/+}$(Fig. 1B, arrowheads). The survival curve indicated that most of $\mathrm{Cldn} 7^{-1-}$ pups died within 9 days after birth (Fig. 1C). Claudin-7 was highly expressed in both small and large intestines in $\mathrm{Cldn} 7^{+/+}$but was completely absent in $C l d n 7^{--}$as confirmed by Western blotting (Fig. 1D). Besides showing salt wasting phenotype as we reported previously, ${ }^{12} \mathrm{Cldn} 7^{-1-}$ mice displayed severe intestinal defects with mucosal ulcerations and villi disruption. Histopathological examinations revealed that on day $\mathrm{P} 0$, there were no differences in villous length and morphology between $\mathrm{Cldn} 7^{+/+}$and $\mathrm{Cldn} 7^{-/-}$small intestines (Fig. 1E, top panel, P0). On day P1, villi in $\mathrm{Cldn7^{-/- }}$ mice were minimally blunted and individual enterocytes sloughed into the intestinal lumen. On day P3, many poorly adhesive enterocytes prematurely sloughed into the lumen as individual cells or in small clusters. On day 5, intestines from $C l d n 7^{-1-}$ mice demonstrated multifocal full thickness mucosal ulcers bordered by strikingly blunted villi. The underlying lamina propria was expanded by mixed inflammatory infiltrates and was covered by a layer of degenerate neutrophils and macrophages. Remaining villi were blunted and the intestinal lumen contained sloughed cells and cellular debris (Fig. 1E, top panel, P5). On day P0, the medial and distal segments of the colon in $C l d n 7^{-1-}$ mice showed the occasional swelling and sloughing of the epithelium into the lumen. On day P1 and P3, epithelial cells lining the colon of $\mathrm{Cldn7^{-1 }}$ mice continued to slough and small mucosal ulcers appeared (Fig. 1E, bottom panel, arrow in P1). An inflammatory infiltrate comprised primarily of macrophages and neutrophils bordered mucosal ulcerations. On day P5, rafts of sloughed epithelial cells were present in the colonic lumen of $\mathrm{Cldn}^{-/-}$mice. Figure $1 \mathrm{~F}$ revealed normal finger-like villi in day $\mathrm{P} 4 \mathrm{Cldn} 7^{+/+}$ small intestines, but a distorted mucosal architecture with short and disorganized villi in day P4 Cldn $7^{-1-}$ small intestines. Similarly, the epithelial lining of $C l d n 7^{-1-}$ large intestine was also disrupted and the surface erosion was obvious when compared with the normal morphology of $\mathrm{Cldn} 7^{+/+}$large intestine (Fig. 1F). Both small and large intestines of $C l d n 7^{-1-}$ pups showed epithelial cell sloughing as indicated by arrows in Figure 1F.

The intestinal epithelial loss was accompanied by increased cell proliferation. As shown in Figure 2, the crypts of day P4 Cldn $7^{-1}$ intestines displayed an abnormally high cell proliferation rate. The mitotic figures in $C l d n 7^{-1-}$ crypt regions can be easily observed (Fig. $2 \mathrm{~A},-/-$, arrows). This high cell proliferation was also confirmed by the BrdU injection experiments. Figures $2 \mathrm{~B}$ and $2 \mathrm{C}$ revealed that the number of dividing cells labeled by BrdU

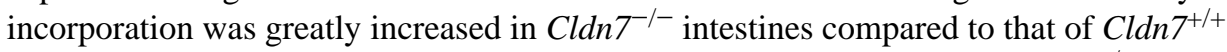
intestines. However, the number of dividing cells did not increase in P0 $\mathrm{Cldn} 7^{-/-}$intestines (Fig. S1). Phosphorylation of histone $\mathrm{H} 3$ was another indication of cell proliferation. Consistent with the BrdU results, the signal of phosphorylated histone $\mathrm{H} 3$ was significantly higher in both small and large intestines of $C l d n 7^{-1-}$ pups than those of $C l d n 7^{+/+}$when analyzed by Western blotting (Fig. 2D). At the same time, cell apoptosis was also increased in $C l d n 7^{-/}$intestines as indicated by the stronger anti-cleaved PARP signal (Fig. 2E). There was no significant difference in cell apoptosis between day P0 Cldn $7^{+/+}$and $C l d n 7^{-1-}$ intestines (Fig. S2). 


\section{Inflammatory responses in $\mathrm{Cldn7^{-/ }}$ mice}

Histological examinations of small intestines from day $\mathrm{P} 4 \mathrm{Cldn} 7^{-1-}$ pups revealed that the lamina propria contained an increased number of leukocytes compared to that in $C l d n 7^{+/+}$, indicating an immune response in $\mathrm{Cldn} 7^{-1-}$ pups (Fig. 3A, the double arrow and arrows in $-/-)$. This observation was confirmed by an antibody recognizing macrophages. The immunostaining signals in both small and large intestines were greatly increased in P4 $C l d n 7^{-/-}$compared to those in $\mathrm{P} 4 \mathrm{Cldn} 7^{+/+}$(Fig. 3B). The macrophages were not increased in P0 $C l d n 7^{-1-}$ intestines (Fig. S3). To determine whether the inflammatory cytokines were upregulated in $C l d n 7^{-1-}$ intestines, qRT-PCR based arrays were performed and then further verified by real-time RT-PCR (Fig. 3C). Indeed, mRNAs of IL-1 $\beta$, IL-8 rb, IL-6, and AP-1, a transcription factor regulating gene transcription, were dramatically increased in day $\mathrm{P} 4$ $C l d n 7^{-I-}$ small and large intestines when compared to those of $C l d n 7^{+/+}$. The TNF $\alpha$ mRNA was also elevated more than 5-fold in $\mathrm{Cldn7^{-1- }}$ intestines. The protein expression levels of NF- $\mathrm{kB}$, c-Jun, c-Fos, and COX-2 were all significantly increased in $\mathrm{Cldn} 7^{-1-}$ intestines, while $\mathrm{p} 38$ and ERK1/2 expressions were not (Fig. 3D). The phosphorylations of NF- $\mathrm{KB}$ and c-Jun in $C l d n 7^{-1-}$ intestines were also greatly elevated, but p38 and ERK1/2 phosphorylation levels were unchanged (Fig. 3E). NF-kB has been identified as one of the key regulators in mucosal inflammation in IBD, and its activation was markedly induced in IBD patients. ${ }^{15-16}$ Increased expression of COX-2 has been reported in many active human IBD patient samples. ${ }^{17} \mathrm{C}$-Jun and c-Fos are the components of AP-1, which is the downstream element of NF- $\mathrm{kB}$. The significant increase of these proteins indicated that mice lacking claudin-7 evoked the intestinal inflammation. Although histological data did not show inflammation in P0 Cldn $7^{-1-}$ intestines, and NF- $\mathrm{KB}$ p 65 subunit and COX-2 were not increased in P0 Cldn $7^{-/-}$intestines, several cytokine mRNA levels, especially the IL-1 $\beta$ mRNA level, were elevated, indicating that the inflammation was initiated at the message level (Fig. S4).

\section{MMP-3 increase in $\mathrm{Cldn7^{-1 }}$ intestines was the direct consequence of claudin-7 deletion}

It has been demonstrated that uncontrolled activation of MMPs can result in tissue injury and inflammation. ${ }^{18}$ In an attempt to investigate the potential mechanism underlying the damage of intestines in $C l d n 7^{-1-}$ mice, we examined a panel of MMPs using a real-time RTPCR approach. We found that MMP-3 mRNA was increased more than 120-fold in P4 $C l d n 7^{-/-}$small intestine compared to that of $C l d n 7^{+/+}$(Fig. 4A). MMP-7 and MMP-8 mRNAs were enhanced 20 to 40 -fold in $C l d n 7^{-1-}$ small intestine. In day P0 intestines, only MMP-3 mRNA level was dramatically increased (Fig. S5 A). At the protein level, MMP-3 and MMP-7 were significantly increased in both small and large intestines of day P4 $C l d n 7^{-/-}$mice (Fig. 4B). Interestingly, MMP-8 protein expression was not elevated in $\mathrm{Cldn7^{-1 }}$ intestines. MMP-7 is secreted as a $28 \mathrm{kDa}$ proenzyme that can be activated in vivo by MMP-3 to form a $19 \mathrm{kDa}$ active MMP-7. ${ }^{19}$ The active form of MMP-3 is at $45 \mathrm{kDa}$. The enzymatic activity of MMP-3 and MMP-7 can be detected by using zymography based on molecular weight shift from latent to activated forms. Figure $4 \mathrm{C}$ showed the casein substrate zymography detecting latent and activated MMPs from tissue lysates of $\mathrm{Cldn7^{+/+ }}$ and $C l d n 7^{-1-}$ intestines. The increased band intensity can be observed at 28 and $45 \mathrm{kDa}$ in $C l d n 7^{-1-}$ small intestine and 19,28 , and $45 \mathrm{kDa}$ in $C l d n 7^{-1-}$ large intestine.

To investigate whether MMPs increase was caused by claudin-7 deletion or by the secondary effect of inflammation, we silenced claudin-7 in T84 intestinal epithelial cell line using a siRNA approach. Under the condition in which claudin-7 expression was largely suppressed by the siRNA against claudin-7 (Fig. 4D, insert), only MMP-3 mRNA was increased almost 3-fold without significant changes in the mRNA levels of MMP-7, MMP-8, and other cytokines (Fig. 4D). The effect of claudin-7 on MMP-3 expression was also examined in HCC827 human lung cancer cells, and similar results were obtained. When 
claudin-7 expression was suppressed in HCC827 cells by claudin-7 specific siRNA, the MMP-3 mRNA and protein levels were significantly increased without changes in the mRNA and protein levels of MMP-7 and MMP-8 (Fig. 4E). We also identified that the MMP-3 level in the culture medium was considerably higher in the cells with claudin-7 knockdown (KD), while the MMP-7 and MMP-8 levels were unchanged in T84 cells (Fig. 4F, left panel). The activity of MMP-3 can be inhibited by a MMP-3 inhibitor (Fig. S5 B). To test our hypothesis that the MMP-7 increase in $\mathrm{Cldn} 7^{-1-}$ intestines was caused by inflammatory response, $\mathrm{TNF} \alpha$ was added to the culture medium. Our results demonstrated that MMP-7 expression was greatly upregulated by the addition of TNF $\alpha$, while MMP-3 and MMP-8 were not in T84 cells (Fig. 4F, right panel). Therefore, we concluded that MMP-3 upregulation in $\mathrm{Cldn7^{-1 }}$ intestines was due to claudin-7 deletion, while MMP-7 increase was caused by inflammatory response.

\section{Deletion of claudin-7 altered integrin $\alpha 2$ expression and localization and disrupted the integrin $\alpha 2$ /claudin-1 protein complex}

$C l d n 7^{-/-}$intestines showed severe epithelial cell sloughing (Fig. 5A, $-/-$, arrowhead). Electron microscopic examination revealed the significant intercellular gaps between adjacent epithelial cells and the loosening between cell and matrix in day P5 $\mathrm{Cldn} 7^{-1-}$ small intestine compared to that of $\mathrm{Cldn} 7^{+/+}$(Fig. 5B, arrowheads and arrows, respectively), while the apical TJs were largely intact in $\mathrm{Cldn} 7^{-1-}$ small intestine (Fig. 5C, arrow). In addition, the desmosome was visualized in both $\mathrm{Cldn} 7^{+/+}$and $\mathrm{Cldn7^{-1 }}$ small intestines (Figure 5C, arrowhead). We found that deletion of claudin-7 did not affect the localization of ezrin, an apical marker protein (Fig. 5D). The localization of basolateral marker Na-K ATPase $\alpha 1$ was also similar in $C l d n 7^{-1-}$ small intestines compared to that of $C l d n 7^{+/+}$(Fig. S6). No TJ leakage was detected in $\mathrm{POCldn7^{-/ }}$ intestines when using biotin as a trace molecule although we sometimes observed the disruption of epithelial barrier function at a later stage of $C l d n 7^{-- \text {, }}$ life, such as day P5 (Fig. S7). The intercellular gap was not observed in P0 $\mathrm{Cldn} 7^{-1-}$ intestines (Fig. S8).

Immunofluorescent light microscopy results showed that claudin-7 was strongly expressed at the basolateral membrane and was partially co-localized with integrin $\alpha 2$ in $C l d n 7^{+/+}$ intestines (Fig. 6A, $+/+$, arrowheads). The distribution of integrin $\alpha 2$ was disorganized in $\mathrm{Cldn} 7^{-1-}$ intestines (Fig. 6A, -l-). At the high magnification, the co-localization of claudin-7 and integrin $\alpha 2$ in $\mathrm{Cldn} 7^{+/+}$intestines were clearly visualized at the basal membrane (Fig. 6B,$+/+$, arrowheads). In $C l d n 7^{-1-}$ intestines, integrin $\alpha 2$ was either forming clusters (Fig. 6B, $-1-$, arrowheads) or moving towards the apical lateral surface (Fig. 6B, -1 - , arrows). Claudin- 1 was also partially co-localized with integrin $\alpha 2$ especially at the basal membrane of crypt region in $\mathrm{Cldn} 7^{+/+}$intestines (Fig. 6C, +/+, arrows). However, in $\mathrm{Cldn} 7^{-1-}$ intestines, the normal localization of integrin $\alpha 2$ and claudin- 1 was disrupted and signal of cluster was observed (Fig. 6C, $-/-$, arrows). Integrin $\alpha 2$ signal was more toward apical lateral membrane than at the basal membrane (Fig. 6C, $-1-$, arrowhead). In contrast, claudin-2 was largely localized at the apical membrane, which did not co-localize with integrin $\alpha 2$ (Fig. 6D, +/+, arrows). Although claudin-7 deletion altered the normal distribution pattern of integrin $\alpha 2$, claudin- 2 was still localized at the apical membrane (Fig. $6 \mathrm{D},-1-$, arrows).

To test our hypothesis that claudin-7 and claudin-1 interacted with integrin $\alpha 2$ in $C l d n 7^{+/+}$ intestines, we performed the biochemical analysis. Western blotting results showed that integrin $\alpha 2$ expression was reduced in both $\mathrm{P} 0$ and $\mathrm{P} 5 \mathrm{Cldn} 7^{-1-}$ intestines, while E-cadherin expression was only decreased in P5 $\mathrm{Cldn}^{-/-}$intestines (Fig. 7A). We also found that the expression of desmoglein was reduced in P5 $\mathrm{Cldn}^{-1-}$ intestines as well (Fig. S9). Coimmunoprecipitation results demonstrated that integrin $\alpha 2$ formed a protein complex with both claudin-7 and claudin-1 in $\mathrm{Cldn} 7^{+/+}$intestines (Fig. 7B, +/+, top and middle panels, 
respectively). However, the interaction between claudin- 1 and integrin $\alpha 2$ was almost diminished in the absence of claudin-7 (Fig. 7B, $-/-$, middle panel). In the immunoprecipitates, integrin $\alpha 2$ was clearly present in both $C l d n 7^{+/+}$and $C l d n 7^{-/-}$tissue lysates (Fig. 7B, bottom panel). In contrast, claudin-2 did not form an immunoprecipitable complex with integrin $\alpha 2$, which suggested that they were not interacting with each other even though integrin $\alpha 2$ was present in the immunoprecipitates (Fig. 7C). Figure 7D showed the expression levels of claudin-1 and claudin-2 in P5 Cldn $7^{+/+}$and $C l d n 7^{-1-}$ intestinal lysates. The expression levels of other claudins were shown in Fig. S10. These results indicated that claudin-7/integrin $\alpha 2 /$ claudin-1 formed an interacting complex in $\mathrm{Cldn} 7^{+/+}$ intestines. Reduction of integrin $\alpha 2$ expression (Fig. 7E, top) and interaction of claudin-7 with integrin $\alpha 2$ (Fig. 7E, bottom) were also confirmed in $C l d n 7^{-1-}$ and $C l d n 7^{+/+}$kidneys. As a negative control, claudin- 4 did not interact with integrin $\alpha 2$ in kidneys (Fig. 7F) because claudin-4 was localized at apical TJ area (data not shown).

\section{Discussion}

This study demonstrated that deletion of claudin-7 led to the disruption of intestinal architecture with mucosal ulcerations, epithelial sloughing, and inflammation. We found that in normal intestines, integrin $\alpha 2$ formed a stable protein complex with claudin-7 and claudin-1. This complex was disrupted in $C l d n 7^{-/-}$intestines, and the expression and localization of integrin $\alpha 2$ were also affected, indicating the importance of claudin-7 in mediating cell-matrix interactions. Deletion of claudin-7 also markedly upregulated the expression levels of MMP-3 and MMP-7 that could cause intestinal tissue damage and inflammation. ${ }^{18,20-21}$ However, we were unable to rescue $C l d n 7^{-1-}$ mice by inhibiting MMP-3 and MMP-7 activities using MMP inhibitors (data not shown). This suggests that MMP-3 and MMP-7 upregulation contributed to the phenotypes of $C l d n 7^{-1-}$ mice, but was not the sole reason causing mucosal damage. We propose that disruption of claudin-7 interaction with integrins/claudin-1/ECM components could be the main driving force leading to the severe intestinal defect that cannot be corrected by MMP inhibitors alone.

MMPs are a family of zinc-dependent endopeptidases and are responsible for the tissue remodeling and the degradation of extracellular matrix components. ${ }^{22}$ Excessive or abnormal expression of MMPs contributes to the pathological processes of tissue injury, organ dysfunction, and chronic disease. ${ }^{23}$ An inflammatory response or bacterial infection could also modulate MMPs expression or their activity. For example, MMP-7 expression was significantly increased in inflamed mucosa after Helicobacter pylori strain infection. ${ }^{24}$ Our results showed that claudin-7 depletion in both intestinal T84 and lung HCC 827 cell lines significantly upregulated MMP-3 expression, while MMP-7 and MMP-8 expression remained unchanged. However, MMP-7 expression was dramatically increased when T84 cells were treated with TNF $\alpha$ (Fig. 4F). This suggests that the increase of MMP-7 expression in $C l d n 7^{-/-}$intestines could be due to the inflammatory response. Indeed, the inflammatory and immune responses were clearly observed in $\mathrm{Cldn}^{-1-}$ intestines (Fig. 3). It is possible that claudin-7 deletion leads to MMP-3 expression and activation, which results in the degradation of extracellular matrix components. This action could induce the production of cytokines and stimulate the immune response. Another alternative explanation could be that claudin-7 deletion also affects the epithelial barrier function. The pathogens and microorganisms could enter the body through the defective barrier. Although our biotin permeability assay did not observe the barrier leakage in $\mathrm{P} 0 \mathrm{Cldn}^{-/-}$intestines, the barrier function was disrupted in P5 Cldn $7^{-/}$intestines (Fig. S7).

Studies suggest that barrier defects contribute to the intestine disease. ${ }^{25-26}$ Recently, it has been reported that p120-catenin is essential for maintenance of intestinal barrier function in mice. ${ }^{27}$ Increased mucosal permeability has also been reported in JAM-A deficient mice. ${ }^{28}$ 
However, the work of Turner's group indicates that the intestinal epithelial barrier dysfunction alone may not be sufficient to cause intestinal diseases in mice. ${ }^{29}$ Although several specific claudin deletion or mutation mouse models are available now, none of them show the intestine defects similar to IBD in humans. ${ }^{30-32}$ Therefore, our $\mathrm{Cldn}^{-1-}$ mouse model provides a unique tool for studying the roles of claudins in intestines and the pathogenesis of IBD-like intestinal disorders. Clearly, claudin-7 functions in intestines are irreplaceable. Although multiple claudins, including claudin-1, -2, -3, -4, and -15 , are expressed in the intestines, none of them are able to compensate for the loss of claudin-7 functions. Since the majority of claudin-7 is localized at the basolateral membrane of epithelial cells, which is different from most of the claudins with the apical localization, claudin-7 mediated cell-matrix interaction is indispensible in the intestines

We found that there was no change at the mRNA levels for integrin $\alpha 2$ and claudins. It is possible that deletion of claudin-7 affects integrin binding to the extracellular components and causes integrin internalization and increased degradation. It is also possible that the increased MMP expression can lead to the degradation of integrin $\alpha 2$ and claudin- 1 since both of them have extracellular domains. Although intercellular gaps were not present in P0 $C l d n 7^{-1-}$ intestines, molecular alterations (increased MMP-3 and reduced integrin $\alpha 2$ ) did occur at the birth of $\mathrm{Cldn}^{-1-}$ mice (Fig. S5 A and Fig. 7A). Certainly, the subsequent inflammatory response and increased TJ permeability contributed to $\mathrm{Cldn} 7^{-1-}$ intestinal phenotypes and escalated the mucosa damage.

Our current study provides the strong evidence that claudin-7 has a non-TJ function mediating cell-matrix interactions. First, claudin-7's major location is at the basolateral membrane compartment; second, claudin-7 interacts with integrin $\alpha 2$ at the basolateral membrane of normal intestinal epithelia; third, TJ morphology is similar in $\mathrm{Cldn7^{+/+ }}$ and $C l d n 7^{-1-}$ intestines, while intercellular gaps below TJs are evident when examined by transmission electron microscopy; last, claudin-7 silencing by siRNA in lung HCC827 cells reduces integrin expression and cell adhesion to the matrix. This defect can be partially rescued by ectopically introducing affected integrin (data not shown). Therefore, $\mathrm{Cldn} 7^{-1-}$ mouse model has provided us with the new opportunity and challenge to investigate the nonTJ functions of claudins and presents a new animal model to study the pathogenesis of intestinal disorders and inflammation.

\section{Supplementary Material}

Refer to Web version on PubMed Central for supplementary material.

\section{Acknowledgments}

We thank Beverly G. Jeansonne and Joani T. Zary for their technical assistance. This work is supported by National Institutes of Health grant HL085752 and ES016888 to Y.H.C.

\section{Abbreviations used in this paper}

$\begin{array}{ll}\text { TJ } & \text { tight junction } \\ \text { IBD } & \text { inflammatory bowel disease } \\ \text { qRT-PCR } & \text { quantitative reverse-transcription polymerase chain reaction } \\ \text { MMP } & \text { matrix metalloproteinase } \\ \text { BrdU } & \text { bromodeoxyuridine } \\ \text { IL } & \text { interleukin }\end{array}$



NF-кB
nuclear factor- $\kappa \mathrm{B}$
TNF
tumor necrosis factor

\section{References}

1. Xavier RJ, Podolsky DK. Unravelling the pathogenesis of inflammatory bowel disease. Nature. 2007; 448:427-34. [PubMed: 17653185]

2. Smalley-Freed WG, Efimov A, Burnett PE, et al. p120-catenin is essential for maintenance of barrier function and intestinal homeostasis in mice. J Clin Invest. 2010; 120:1824-35. [PubMed: 20484816]

3. Furuse M, Tsukita S. Claudins in occluding junctions of humans and flies. Trends Cell Biol. 2006; 16:181-8. [PubMed: 16537104]

4. Tamura A, Kitano Y, Hata M, et al. Megaintestine in claudin-15-deficient mice. Gastroenterology. 2008; 134:523-34. [PubMed: 18242218]

5. Weber CR, Turner JR. Inflammatory bowel disease: is it really just another break in the wall? Gut. 2007; 56:6-8. [PubMed: 17172583]

6. Zeissig S, Burgel N, Gunzel D, et al. Changes in expression and distribution of claudin 2, 5 and 8 lead to discontinuous tight junctions and barrier dysfunction in active Crohn's disease. Gut. 2007; 56:61-72. [PubMed: 16822808]

7. Hou J, Paul DL, Goodenough DA. Paracellin-1 and the modulation of ion selectivity of tight junctions. J Cell Sci. 2005; 118:5109-18. [PubMed: 16234325]

8. Van Itallie CM, Anderson JM. Claudins and epithelial paracellular transport. Annu Rev Physiol. 2006; 68:403-29. [PubMed: 16460278]

9. Alexandre MD, Lu Q, Chen YH. Overexpression of claudin-7 decreases the paracellular Clconductance and increases the paracellular Na+ conductance in LLC-PK1 cells. J Cell Sci. 2005; 118:2683-93. [PubMed: 15928046]

10. Gonzalez-Mariscal L, Namorado Mdel C, Martin D, et al. The tight junction proteins claudin-7 and -8 display a different subcellular localization at Henle's loops and collecting ducts of rabbit kidney. Nephrol Dial Transplant. 2006; 21:2391-8. [PubMed: 16766545]

11. Mendoza-Rodriguez CA, Gonzalez-Mariscal L, Cerbon M. Changes in the distribution of ZO-1, occludin, and claudins in the rat uterine epithelium during the estrous cycle. Cell Tissue Res. 2005; 319:315-30. [PubMed: 15558325]

12. Tatum R, Zhang Y, Salleng K, et al. Renal salt wasting and chronic dehydration in claudin-7deficient mice. Am J Physiol Renal Physiol. 2010; 298:F24-34. [PubMed: 19759267]

13. Dussault AA, Pouliot M. Rapid and simple comparison of messenger RNA levels using real-time PCR. Biol Proced Online. 2006; 8:1-10. [PubMed: 16446781]

14. Fox JG, Li X, Cahill RJ, et al. Hypertrophic gastropathy in Helicobacter felis-infected wild-type C57BL/6 mice and p53 hemizygous transgenic mice. Gastroenterology. 1996; 110:155-66. [PubMed: 8536852]

15. Atreya I, Atreya R, Neurath MF. NF-kappaB in inflammatory bowel disease. J Intern Med. 2008; 263:591-6. [PubMed: 18479258]

16. Pasparakis M. Regulation of tissue homeostasis by NF-kappaB signalling: implications for inflammatory diseases. Nat Rev Immunol. 2009; 9:778-88. [PubMed: 19855404]

17. Jupp J, Hillier K, Elliott DH, et al. Colonic expression of leukotriene-pathway enzymes in inflammatory bowel diseases. Inflamm Bowel Dis. 2007; 13:537-46. [PubMed: 17230539]

18. Parks WC, Wilson CL, Lopez-Boado YS. Matrix metalloproteinases as modulators of inflammation and innate immunity. Nat Rev Immunol. 2004; 4:617-29. [PubMed: 15286728]

19. Imai K, Yokohama Y, Nakanishi I, et al. Matrix metalloproteinase 7 (matrilysin) from human rectal carcinoma cells. Activation of the precursor, interaction with other matrix metalloproteinases and enzymic properties. J Biol Chem. 1995; 270:6691-7. [PubMed: 7896811]

20. Pender SL, Tickle SP, Docherty AJ, et al. A major role for matrix metalloproteinases in T cell injury in the gut. J Immunol. 1997; 158:1582-90. [PubMed: 9029093] 
21. Sorokin L. The impact of the extracellular matrix on inflammation. Nat Rev Immunol. 10:712-23. [PubMed: 20865019]

22. Wilson CL, Matrisian LM. Matrilysin: an epithelial matrix metalloproteinase with potentially novel functions. Int J Biochem Cell Biol. 1996; 28:123-36. [PubMed: 8729000]

23. Manicone AM, McGuire JK. Matrix metalloproteinases as modulators of inflammation. Semin Cell Dev Biol. 2008; 19:34-41. [PubMed: 17707664]

24. Crawford HC, Krishna US, Israel DA, et al. Helicobacter pylori strain-selective induction of matrix metalloproteinase-7 in vitro and within gastric mucosa. Gastroenterology. 2003; 125:1125-36. [PubMed: 14517796]

25. Bruewer M, Samarin S, Nusrat A. Inflammatory bowel disease and the apical junctional complex. Ann N Y Acad Sci. 2006; 1072:242-52. [PubMed: 17057204]

26. Turner JR. Intestinal mucosal barrier function in health and disease. Nat Rev Immunol. 2009; 9:799-809. [PubMed: 19855405]

27. Smalley-Freed WG, Efimov A, Burnett PE, et al. p120-catenin is essential for maintenance of barrier function and intestinal homeostasis in mice. J Clin Invest. 120:1824-35. [PubMed: 20484816]

28. Laukoetter MG, Nava P, Lee WY, et al. JAM-A regulates permeability and inflammation in the intestine in vivo. J Exp Med. 2007; 204:3067-76. [PubMed: 18039951]

29. Su L, Shen L, Clayburgh DR, et al. Targeted epithelial tight junction dysfunction causes immune activation and contributes to development of experimental colitis. Gastroenterology. 2009; 136:551-63. [PubMed: 19027740]

30. Gow A, Southwood CM, Li JS, et al. CNS myelin and sertoli cell tight junction strands are absent in Osp/claudin-11 null mice. Cell. 1999; 99:649-59. [PubMed: 10612400]

31. Furuse M, Hata M, Furuse K, et al. Claudin-based tight junctions are crucial for the mammalian epidermal barrier: a lesson from claudin-1-deficient mice. J Cell Biol. 2002; 156:1099-111. [PubMed: 11889141]

32. Nitta T, Hata M, Gotoh S, et al. Size-selective loosening of the blood-brain barrier in claudin-5deficient mice. J Cell Biol. 2003; 161:653-60. [PubMed: 12743111] 
A

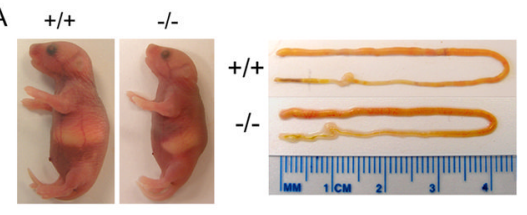

C

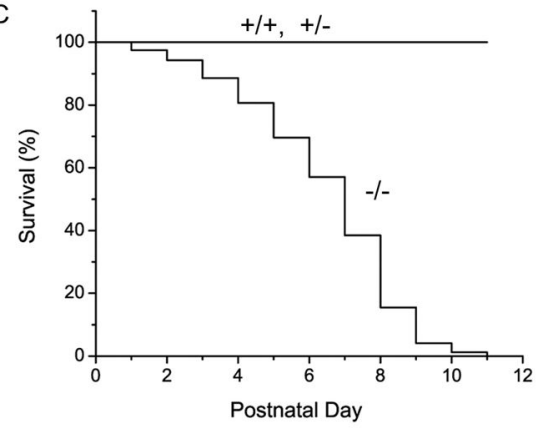

B

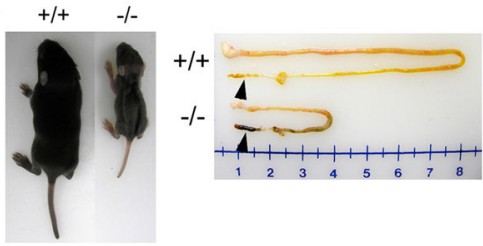

D

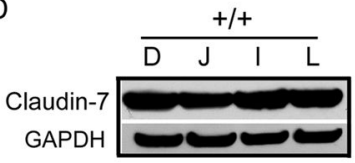

E $\mathrm{PO}+/+\quad \mathrm{PO}-/-$ P1 -/-
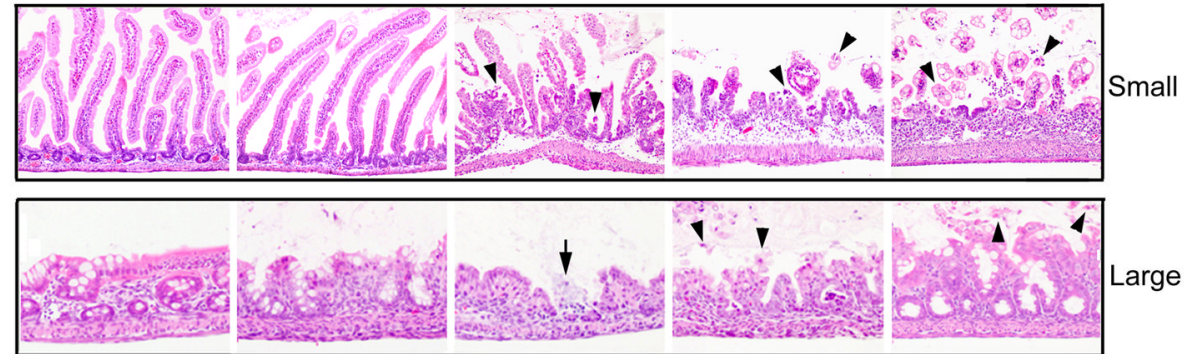

$\mathrm{F}$
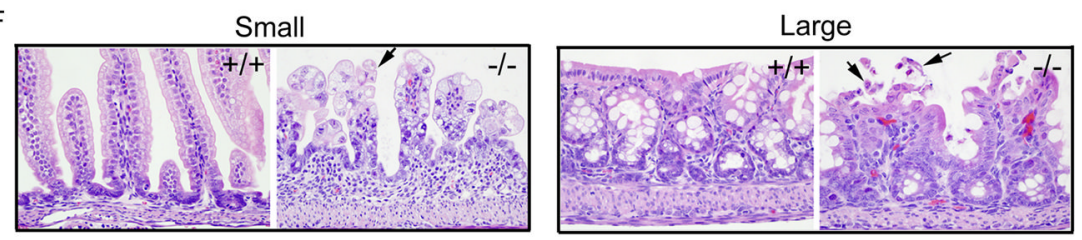

Figure 1.

Deletion of claudin-7 disrupted the intestinal epithelial structure in mice. (A) New born (P0) $C l d n 7^{+/+}$and $C l d n 7^{-/-}$pups and their isolated intestines. (B) Size comparison of one-week old $C l d n 7^{+/+}$and $C l d n 7^{-/-}$littermates and their dissected intestinal tracts. The arrowheads indicated the normal shaped stool in $\mathrm{Cldn} 7^{+/+}$large intestine, but melena in $\mathrm{Cldn} 7^{-/-}$. (C) Survival curve of $\mathrm{Cldn} 7^{-1-}$ mice. About $95 \%$ of $279 \mathrm{Cldn} 7^{-1-}$ mice died within 10 days after birth. (D) Immunoblotting revealed the similar amount of claudin-7 expression in $C l d n 7^{+/+}$duodenum (D), jejunum (J), ileum (I), and large intestine (L) and the absence of claudin-7 in $C l d n 7^{-1-}$ intestines. (E) Light micrographs of H\&E stained small and large intestines from P0, P1, P3, and P5 Cldn $7^{+/+}$and $C l d n 7^{-/-}$pups. The sections showed the normal $C l d n 7^{+/+}(+/+)$and timely deteriorated $C l d n 7^{-/-}(-/-)$mucosa. The arrowheads indicated the sloughing epithelial cells. The arrow indicated the site of ulcer. (F) Enlarged H\&E stained images to show that the epithelial cells lining the villi in P4 Cldn $7^{-1-}$ mice lost their polarity and culomnar orientation and became polygonal and markedly swollen. The arrows pointed to the sloughing epithelial cells in small and large $C l d n 7^{-1-}$ intestines. Magnifications: $(\mathbf{E})$ top, $\times 200$; bottom, $\times 400 ;(\mathbf{F}) \times 600$. 

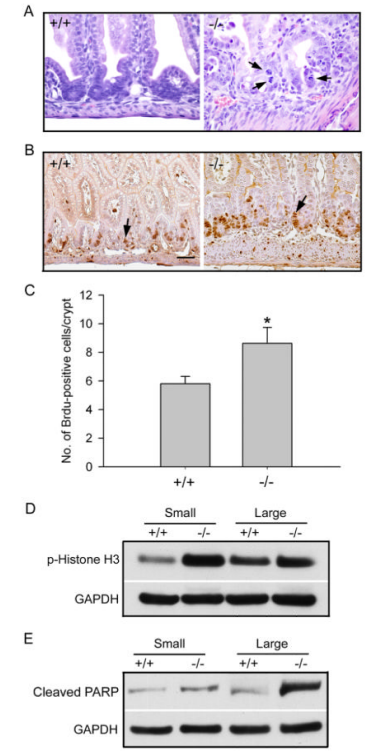

Figure 2.

Increased cell proliferation in $C l d n 7^{-1-}$ intestines. (A) H\&E staining of small intestines from day P4 Cldn $7^{+/+}$and $C l d n 7^{-/-}$pups. Arrows indicated the mitotic figures in $C l d n 7^{-1-}$ crypt regions. Magnification: $\times 600$. (B) $C l d n 7^{+/+}$and $C l d n 7^{-/-}$were injected with BrdU (50mg/ $\mathrm{kg}$ ) and sacrificed $2 \mathrm{~h}$ after injection. Sections of the small intestine were stained with antiBrdU antibody. Bar: $40 \mu \mathrm{m}$. (C) Statistical analysis revealed the increased numbers of mitotic cells in $C l d n 7^{-/-}$small intestines (-/-) compared to those of $C l d n 7^{+/+}(+/+)$. Data was obtained from three independent experiments. (D) and (E) Intestinal lysates from $\mathrm{Cldn} 7^{+/+}$and $\mathrm{Cldn} 7^{-/-}$pups were immunoblotted with anti-phospho-Histone H3 and anticleaved PARP antibodies. GAPDH served as a protein loading control. 


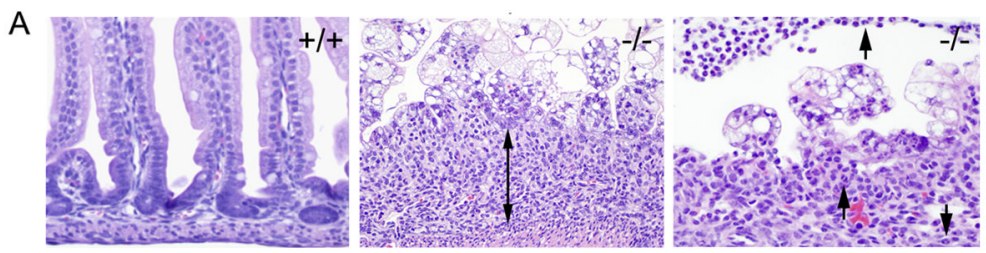

B
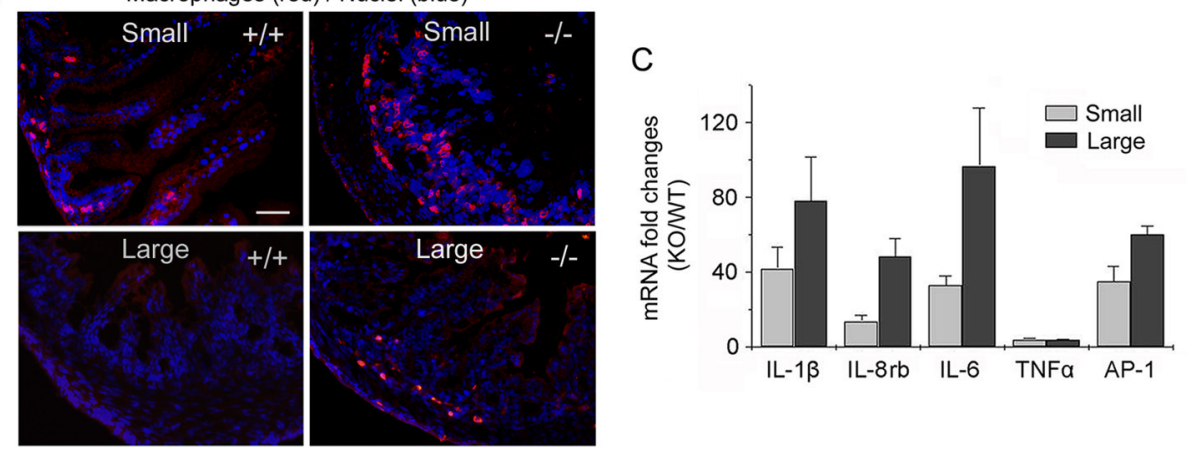

D

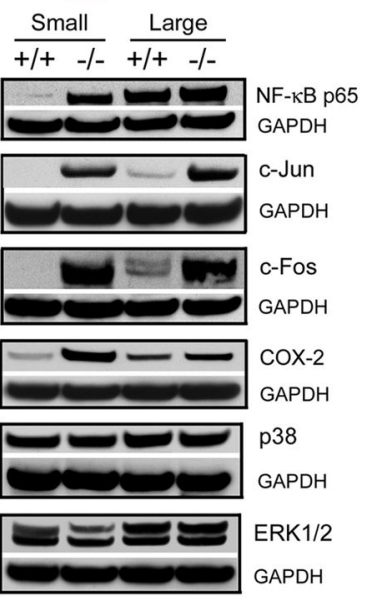

E

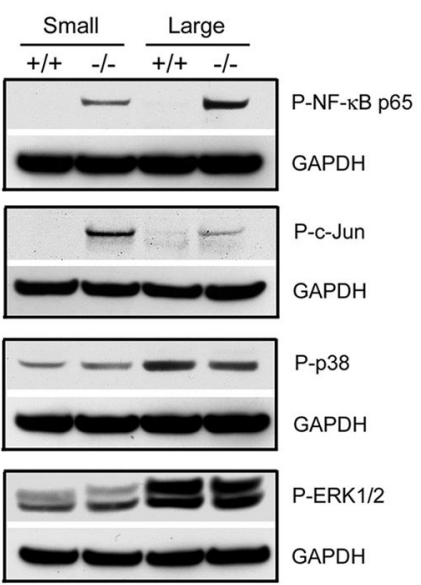

Figure 3.

The inflammatory response in $C l d n 7^{-/-}$intestines. (A) Light micrographs of H\&E stained small intestines from day $\mathrm{P} 4 \mathrm{Cldn} 7^{+/+}(+/+)$and $C l d n 7^{-/-}(-/-)$pups. The $C l d n 7^{-/-}$section showed the large amount of mixed inflammatory cells in the lamina propria area (double arrow in $-/-$ ). At the high magnification, neutrophils can be observed (arrows in $-/-$ ). Magnifications: left and middle images, $\times 400$; right image, $\times 600$. (B) Frozen sections of intestinal tissues were stained with an antibody recognizing macrophages and DAPI. Bar: 20 $\mu \mathrm{m}$. (C) The mRNA levels of cytokines and AP-1 were greatly increased in $\mathrm{Cldn} 7^{-/-}$ intestines measured by qRT-PCR. This increase was expressed as fold changes when comparing $C l d n 7^{-/-}(\mathrm{KO})$ values to that of $C l d n 7^{+/+}$(WT) after they were normalized to the GAPDH value. The data were from five independent experiments. (D) The expression levels of NF- $\kappa \mathrm{B}$ p65, c-Jun, c-Fos, and COX-2 were greatly increased in $C l d n 7^{-/}$intestines by immunoblotting analysis. The p38 and ERK1/2 expressions were not enhanced in $\mathrm{Cldn}^{-1-}$ intestines. GAPDH served as a loading control. (E) The immunoblotting results showed that $\mathrm{NF}-\kappa \mathrm{B}$ p65 and c-Jun proteins in $\mathrm{Cldn} 7^{-1-}$ intestines were highly phosphorylated using antiphospho-NF- $\mathrm{B}$ p65 and anti-phospho-c-Jun antibodies. The levels of phospho-p38 and phospho-ERK1/2 did not have significant changes between $\mathrm{Cldn} 7^{+/+}$and $\mathrm{Cldn} 7^{-/-}$ intestines. 

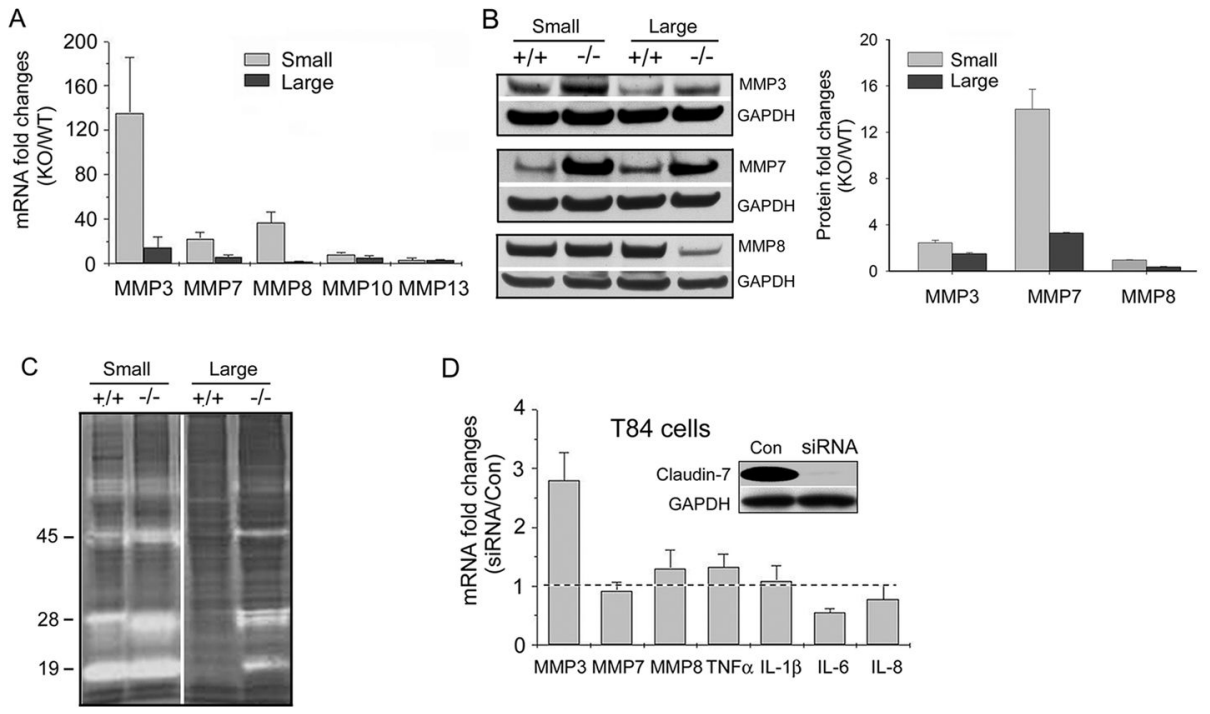

D

E
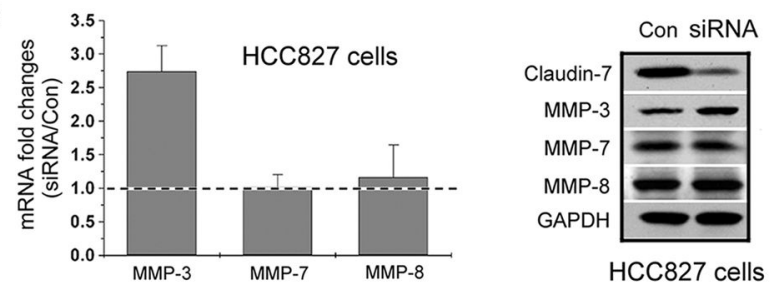

F
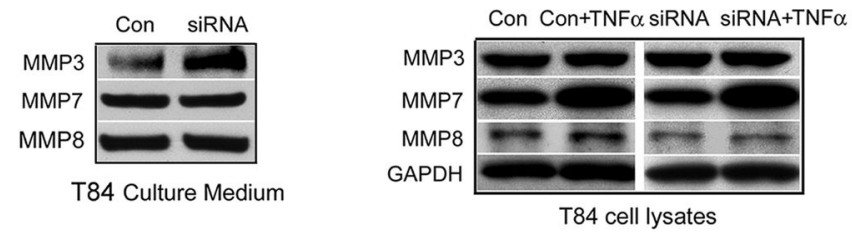

Figure 4.

Upregulation of MMPs in day P4 $C l d n 7^{-/-}$intestines. (A) The mRNA levels of MMPs in $C l d n 7^{-1-}$ intestines were greatly increased by qRT-PCR analysis. Each data point was obtained from five independent experiments. (B) MMP-3 and MMP-7 expressions, but not MMP-8, were significantly enhanced in $C l d n 7^{-1-}$ intestines by immunoblotting examinations. The band intensity measurements (present as KO/WT) were shown on the right. (C) Increased enzymatic activities of MMPs in $C l d n 7^{-1-}$ intestines were revealed by casein zymography gel. (D) Upregulation of MMP-3 in T84 epithelial cells with claudin-7 knockdown (KD). The insert showed the claudin-7 KD by $50 \mu \mathrm{M}$ siRNA against claudin-7 for $72 \mathrm{~h}$. The scramble siRNA was used in control cells (Con). Only MMP-3 mRNA was significantly increased with claudin-7 KD by qRT-PCR analysis. The data represented the mean values of five independent experiments and were normalized with endogenous GAPDH values. The dotted line represents no change when the ratio equals 1. (E) Real-time qRT-PCR data also showed a significant increase of MMP-3 mRNA (left) and protein (right) in human lung HCC827 cells with claudin-7 KD by specific siRNA. (F) Culture media were collected from T84 cells treated with scramble siRNA (Con) or specific siRNA against claudin-7 (siRNA). Immunoblotting displayed the increased level of MMP-3, but not MMP-7 and MMP-8 (left). MMP-7 expression was greatly increased in both control and 
claudin-7 KD cells after the addition of cytokine $\mathrm{TNF} \alpha(20 \mathrm{ng} / \mathrm{mL})$ to the culture medium for $24 \mathrm{~h}$ (right). 


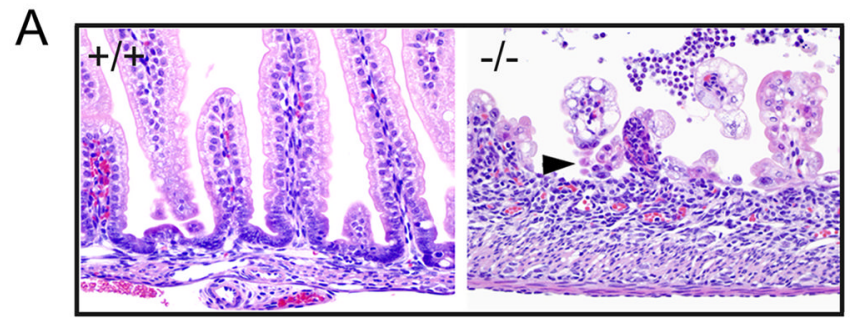

B
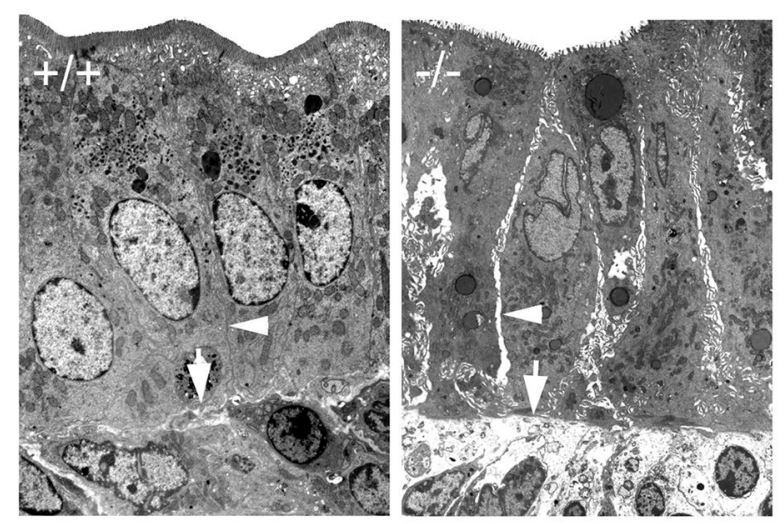

C
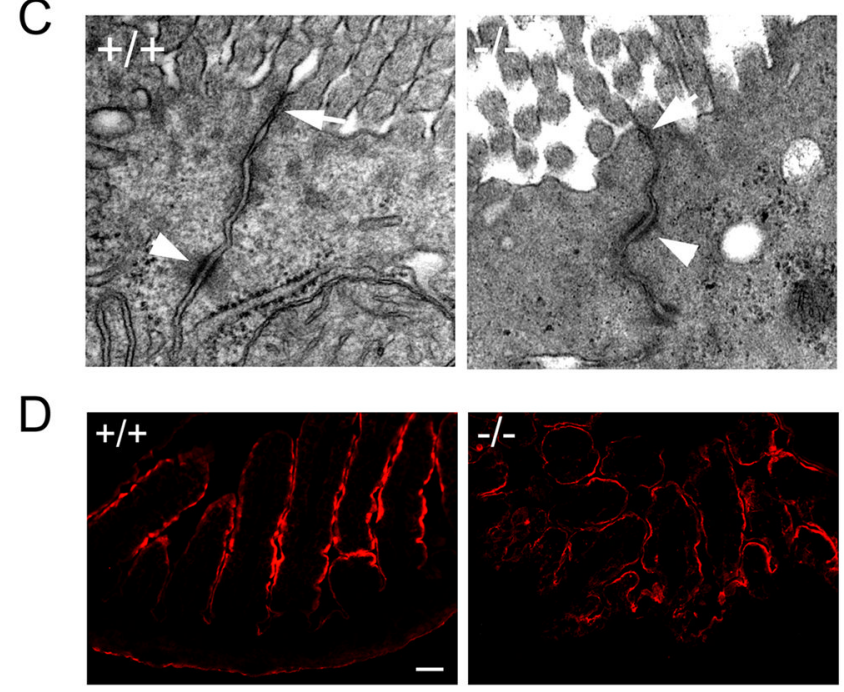

Figure 5.

Disruption of cell-cell adhesion in $C l d n 7^{-/-}$intestines. (A) Light micrographs of $\mathrm{H} \& \mathrm{E}$ stained day P5 Cldn $7^{+/+}(+/+)$and $C l d n 7^{-1-}(-/-)$ small intestines. The arrowhead in (-/-) pointed to the sloughing epithelial cells. Magnification: $\times 400$. (B) Electron micrographs of day P5 $\mathrm{Cldn} 7^{+/+}(+/+)$and $C l d n 7^{-/-}(-/-)$small intestines. The arrowhead in (-/-) pointed to the intercellular gap along the $C l d n 7^{-1-}$ lateral membrane compared to that of $C l d n 7^{+/+}$ $(+/+$, arrowhead). The arrow in $(-/-)$ revealed the loosening of cell-matrix connection in $C l d n 7^{-/-}$versus the close contact between cell and matrix in $C l d n 7^{+/+}$intestines $(+/+$, arrow). (C) The arrows in $(+/+)$ and $(-/-)$ pointed to the apical TJ. The arrowheads indicated the desmosome. Magnifications: $(\mathbf{B}), \times 5,000 ;(\mathbf{C}), \times 50,000$. (D) Frozen sections of $\mathrm{Cldn} 7^{+/+}(+/+)$and $C l d n 7^{-/-}(-/-)$small intestines were immunostained with an apical protein marker, Ezrin. Bar: $40 \mu \mathrm{m}$. 

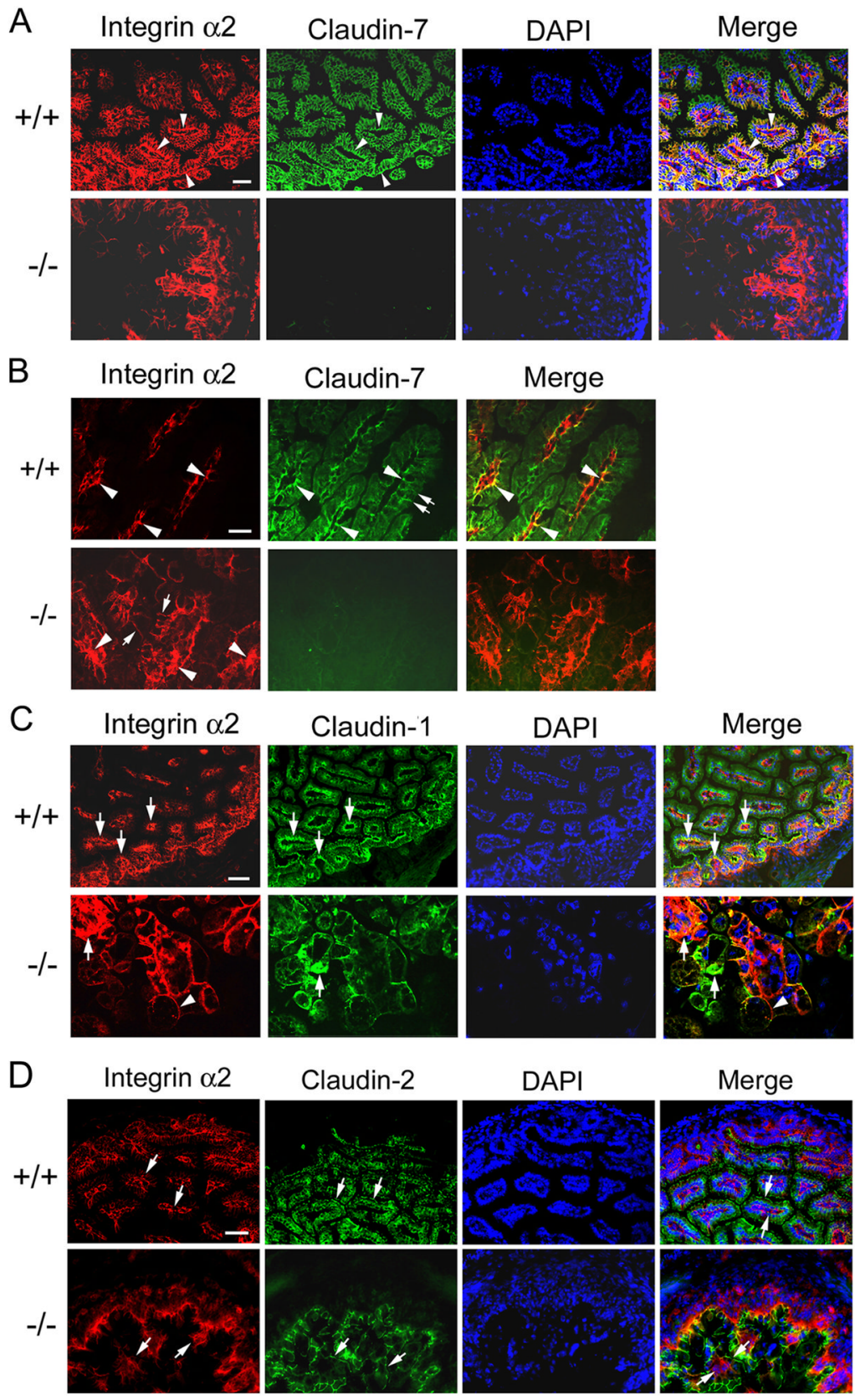

Figure 6.

Disruption of integrin $\alpha 2$ localization in $C l d n 7^{-/-}$intestines. (A) Arrowheads in (+/+) showed examples of double immunostaining signals where claudin-7 and integrin $\alpha 2$ were co-localized at the basal membrane. Integrin $\alpha 2$ signals were disorganized in (-/-). Bar: 40 $\mu \mathrm{m}$. (B) High magnification to show the co-localization of claudin-7 and integrin $\alpha 2$ in $\mathrm{Cldn} 7^{+/+}$(arrowheads in $+/+$) and disorganized integrin $\alpha 2$ signal in $C l d n 7^{-/-}$intestines (arrowheads in $-/-$ ). The double arrows in (+/+) indicated the apical surface of the villus and arrows in $(-/-)$ pointed to the integrin $\alpha 2$ signal moving towards apical lateral surface. Bar: $20 \mu \mathrm{m}$. (C) Integrin $\alpha 2$ and claudin-1 were partially co-localized at the basolateral region of $\mathrm{Cldn} 7^{+/+}$villi (arrows in $+/+$). Arrows in (-/-) indicated the clustered signals of integrin $\alpha 2$ and claudin- 1 , while the arrowhead pointed to integrin $\alpha 2$ moving towards the 
apical lateral surface in the absence of claudin-7. Bar: $40 \mu \mathrm{m}$ for the top panel and $20 \mu \mathrm{m}$ for the bottom panel. (D) Claudin- 2 and integrin $\alpha 2$ were not co-localized since claudin- 2 signal was largely localized at the apical membrane even in the absence of claudin-7 (claudin-2, arrows). Integrin $\alpha 2$ signal (integrin $\alpha 2$, arrows) was mainly in the basolateral region in $\mathrm{Cldn} 7^{+/+}$, but was disrupted in $\mathrm{Cldn} 7^{-/-}$. Arrows in merge images pointed to claudin-2 (top) and integrin $\alpha 2$ (bottom) signals. Bar: $20 \mu \mathrm{m}$. 
A

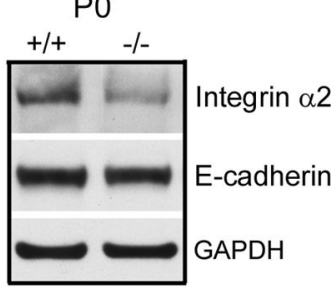

C

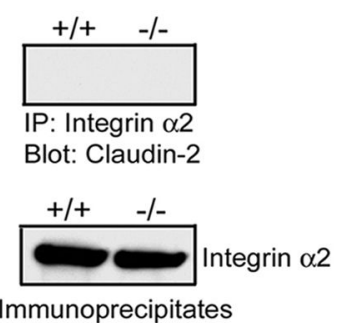

E

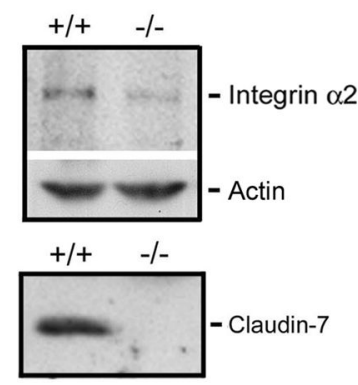

IP: Integrin $\alpha 2$ Blot: Claudin-7
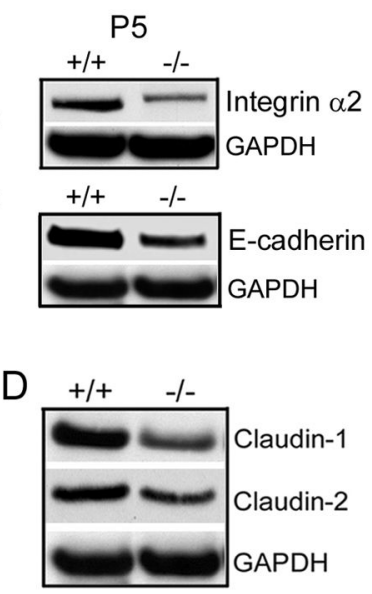

Tissue lysates

$\mathrm{F}$

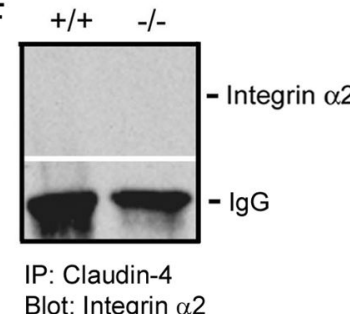

B
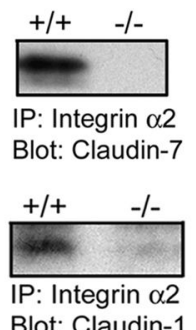

Blot: Claudin-1
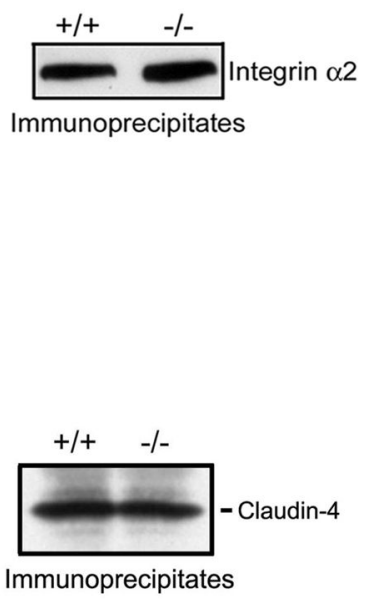

Immunoprecipitates

Figure 7.

Disruption of integrin $\alpha 2 /$ claudin-1 complex in $C l d n 7^{-1-}$ intestines. (A) Immunoblotting results showed that integrin $\alpha 2$ expression, but not E-cadherin, was decreased in P0 $C l d n 7^{-1-}$ intestines (left), while both integrin $\alpha 2$ and E-cadherin signals were reduced in P5 $C l d n 7^{-1-}$ intestines (right). (B) Claudin-7 was co-immunoprecipitated with integrin $\alpha 2$ in $C l d n 7^{+/+}$(top). Claudin-1 also formed a complex with integrin $\alpha 2$ in $C l d n 7^{+/+}$, but this complex was disrupted in $\mathrm{Cldn}^{-1-}$ (middle). The co-immunoprecipitation experiments were working since integrin $\alpha 2$ was present in the immunoprecipitates (bottom). (C) Claudin-2 did not interact with integrin $\alpha 2$ (top) even though integrin $\alpha 2$ was present in the immunoprecipitates (bottom). (D) Expression levels of claudin-1 and claudin-2 in $\mathrm{Cldn} 7^{+/+}$ and $C l d n 7^{-1-}$ intestines. (E) Interaction of claudin-7 with integrin $\alpha 2$ in $C l d n 7^{+/+}$kidneys. Claudin-7 deletion also led to the reduced integrin $\alpha 2$ expression in kidneys (top). Claudin-7 formed an immunoprecipitable protein complex with integrin $\alpha 2$ in $C l d n 7^{+/+}$kidneys (bottom). (F) Integrin $\alpha 2$ did not interact with claudin- 4 (left) even though claudin- 4 was present in the immunoprecipitates (right). 